\title{
An Application of Fuzzy Integrated Model in Green Supplier Selection
}

\author{
Alptekin Ulutaş $\mathbb{D}^{1},{ }^{1}$ Ayşe Topal, ${ }^{2}$ and Rim Bakhat ${ }^{3}$ \\ ${ }^{1}$ Department of International Trade and Logistics, Faculty of Economics and Administrative Sciences, \\ Sivas Cumhuriyet University, Sivas 58140, Turkey \\ ${ }^{2}$ Department of Business, Faculty of Economics and Administrative Sciences, Nigde Omer Halisdemir University, Nigde 51240, Turkey \\ ${ }^{3}$ Department of Economics and Social Sciences, University Abdel Malek Essadi, Tangier-Tétouan 93030, Morocco
}

Correspondence should be addressed to Alptekin Ulutaş; aulutas@cumhuriyet.edu.tr

Received 7 March 2019; Accepted 8 April 2019; Published 18 April 2019

Academic Editor: Anna M. Gil-Lafuente

Copyright (C) 2019 Alptekin Ulutaş et al. This is an open access article distributed under the Creative Commons Attribution License, which permits unrestricted use, distribution, and reproduction in any medium, provided the original work is properly cited.

\begin{abstract}
"Sustainability" term has not only become increasingly important globally for individual companies, but also become important for whole supply chains. The selection of supplier is a significant decision for the sustainability of supply chains. Literature review revealed that supplier selection is made traditionally based on economic attributes which are insufficient for sustainability of supply chains as sustainability requires taking economic, environmental, and social issues into account. For this purpose, this paper proposes determining the green supplier selection attributes and then developing a methodology for assessment and ranking of green suppliers based on determined attributes. The first contribution of this study is to propose a novel method, which is FROV (fuzzy extension of range of value) to literature. The latter is to utilize fuzzy extension of preference selection index (FPSI) to identify the weights of attributes. The third is to develop a novel fuzzy multiattribute decision-making model consisting of FPSI and FROV to determine the best supplier for a Turkish textile company.
\end{abstract}

\section{Introduction}

"Sustainable Development" has become increasingly important globally in recent decades. The World Commission on Environment and Development [1] explained "Sustainable Development" as supplying resources for meeting the needs of people currently living without making a significant impact on the resources needed for people living in the future, in Our Common Future report. It is suggested in the report that effective long-term development will be successful if economic, environmental, and social concerns will be taken into consideration.

Numerous studies have shown that, with the entry of sustainability into plans and policies, meeting environmental and social goals together with economic goals has been important not only for government sector but also for private sector such as construction [2-5], fisheries [6-11], mining [12-17], and transportation [18-24].

Despite the studies above discussing sustainability in the scale of individual companies, Vachon and Klassen [51] suggested that the environmental management should not be restricted to the individual companies alone and must go further to the whole supply chain involving all companies throughout the entire life of product. For this purpose, the perception of GSCM (Green Supply Chain Management) appeared in the literature during the 1990s when competition had an increasing trend [52]. GSCM is defined as incorporating environmental or green concerns into the supply chain processes beginning from design of the product to recycling of the goods at the end of its life [53].

Traditionally, supplier selection has a crucial role in supply chains as it contributes to increasing product quality and customer satisfaction [54]. It has become more important and complex with recent trends, sustainability development, and GSCM. Therefore, developing a model for selecting green suppliers is necessary for maintaining sustainability in a supply chain. The process of supplier selection in traditional supply chains has been named as green supplier selection in GSCM and became a central part of GSCM [55]. 
TABLE 1: The most common used economic attributes in supplier selection.

\begin{tabular}{lc}
\hline Quality & Financial Performance \\
Delivery (Time) & Management and Organization \\
Price & Production Facilities and Capacity \\
Flexibility & Reliability \\
Technical Capability & Long-term Relationship \\
\hline Sources: $[25-37]$. &
\end{tabular}

For this purpose, this paper proposes determining the green attributes for the selection of supplier and then developing a model for assessing and ordering of green suppliers based on determined attributes. Three contributions have been made into the literature by this paper. The first is to propose a novel model, which is fuzzy extension of range of value (FROV) to literature. Generally, fuzzy extension of preference selection index (FPSI) has not been applied to acquire attribute weights, so the second is to utilize FPSI to identify the weights of attributes. The third is to develop a novel fuzzy multiattribute decision-making (MADM) model consisting of FPSI and FROV to address supplier selection problem for a Turkish textile company.

The rest of the paper is organized as follows. After the introduction, the attributes used for selecting green supplier in the literature are reviewed in Section 2. Then, the novel model is explained in detail in Section 3. In Section 4, the results are presented and the results of FROV are compared with the results of other fuzzy MADM, which are fuzzy additive ratio assessment (ARAS) [56], fuzzy multiple objective optimization on the basis of ratio analysis plus full multiplicative form (MULTIMOORA) [57], fuzzy complex proportional assessment (COPRAS) [58], and fuzzy grey relational analysis (GRA) [59]. In Section 5, a sensitivity analysis is done in order to observe the changing of the results with respect to the changing of attribute weights. In Section 6, a brief conclusion is presented.

\section{Literature Review}

In the literature review section, attributes utilized for green supplier selection in the literature have been reviewed to determine which attributes to be used in the model (Section 3). There are three groups of attributes in the literature used for green supplier selection: economic, environmental, and social. Environmental and social attributes are addressed as green attributes in this section.

2.1. Economic Attributes. Economic attributes have been used commonly for a long time in supplier selection before sustainability introduced to supply chain management [25]. The economic attributes mostly used for supplier selection were summarized in Table 1 .

Due to the profit maximization objectives of firms, price attribute was once the most popular economic attribute used in supplier selection $[26,27]$. However, it was shown that price attribute alone is insufficient for supplier selection problems in the literature review conducted by Ho et al. [28]. The review presented that quality, delivery, management, technology, relationship, and flexibility attributes are also significant in supplier selection besides price attribute. Financial performance attribute is deemed significant by Büyüközkan and Çifçi [29] and Dickson [30] as it shows the stability and thus the continuity of supplier firms. Dickson [30] presented the importance of production capacity in addition to the attributes mentioned above. Kannan et al. [31] indicated that reliability attribute is among most common used traditional attributes in the literature.

As it is seen in the literature, economic attributes are the main focus for traditional supplier selection. However, recent changes in the economy and politics such as increasing competition and foreign trade, consumers, and governments giving more importance to sustainability therefore required environmental and social attributes to be included in supplier selection.

2.2. Green Attributes. Several studies in the literature addressed the green attributes (social and environmental) in supplier selection problems. Table 2 summarizes the green attributes used in the literature.

Noci [38] presented a supplier selection model based on environmental performance of suppliers. It was claimed that increasing concerns about environmental performance of corporates will lead the corporates to choose suppliers based on an environmental viewpoint. Handfield et al. [39] presented an analytic hierarchy process (AHP) based supplier selection model to be used in the selection of suppliers based on environmental responsibility attribute. Humphreys et al. [40] introduced a supplier selection decision-making model by using Knowledge-Based System (KBS) which used qualitative and quantitative environmental attributes.

Lee et al. [32] developed an integrated model including fuzzy AHP and Delphi methods to choose the most appropriate green suppliers for corporations. Kuo et al. [33] created green supplier selection model by integrating the artificial neural network (ANN), the data envelopment analysis (DEA), and analytic network process (ANP).

Büyüközkan and Çifçi [41] conducted a study to evaluate the sustainability of suppliers with ANP. Kannan et al. [42] selected suppliers based on economic and environmental attributes in their model integrating fuzzy AHP, fuzzy technique for order preference by similarity to ideal solution (TOPSIS), multiobjective linear programming, and fuzzy logic. Hashemi et al. [43] suggested a comprehensive green supplier selection model by using ANP, the traditional GRA, and both economic and environmental attributes. Rostamzadeh et al. [44] presented a model for evaluating green suppliers based on quantitative data by using fuzzy sets 
TABLE 2: Green attributes used in supplier selection.

\section{ATTRIBUTES}

(i) Using green technologies

(ii) Environmental efficiency

(iii) Supplier's green image

(iv) Reverse logistics (recycling, remanufacturing, reusing)

(v) Reducing activities

(i) Pollution level

(ii) Waste management

(iii) Noise

(iv) Resource consumption (energy, material, water)

(i) Green packaging and labelling

(ii) Green transportation

(iii) Green product design

(iv) Green procurement

(v) Green warehousing

(vi) Green innovation and R\&D

(vii) Green stock politics

(i) Occupational health and safety systems,

(ii) Social responsibility

(iii) Employees' interests and rights

(iv) The stakeholders' rights

Sources: [32-34, 38-50].

TABLE 3: Linguistic and fuzzy performance ratings.

\begin{tabular}{lc}
\hline Linguistic Performance Ratings & Fuzzy Performance Ratings \\
\hline Very Strong & $(7,9,10)$ \\
Strong & $(5,7,9)$ \\
Moderate & $(3,5,7)$ \\
Weak & $(1,3,5)$ \\
Very Weak & $(0,1,3)$
\end{tabular}

theory and the Vlsekriterijumska Optimizacija I Kompromisno Resenje (VIKOR) method.

Awasthi and Kannan [45] developed an integrated decision approach to analyse and select development programs for green suppliers by using a fuzzy nominal group technique (NGT) and VIKOR. Uygun and Dede [46] provided an integrated fuzzy model including fuzzy decision-making trial and evaluation laboratory (DEMATEL) method, ANP, and fuzzy TOPSIS to analyse green performances of companies in supply chains. Chen et al. [60] proposed fuzzy AHP and fuzzy TOPSIS to solve green supplier selection problem. Pang et al. [61] proposed a fuzzy grey model to address green supplier selection problem. Guo et al. [47] presented a framework including the triple bottom line principle and fuzzy axiomatic design $(\mathrm{AD})$ technique to solve green supplier selection problem in global apparel industry. Luthra et al. [34] provided a framework selecting suppliers in accordance with sustainability by using a model integrating AHP and VIKOR methods. They identified 22 economic, environmental, and social attributes. Wang et al. [48] developed a framework for selecting green suppliers by using the cloud model and qualitative flexible multiple criteria method (QUALIFLEX) with the economic and environmental attributes.

Vahidi et al. [49] developed a novel possibilisticstochastic model for the selection of sustainable suppliers. It showed that sustainability is significant in terms of reducing supply costs. Yu et al. [50] presented a model for selecting green suppliers based on carbon footprints. Yucesan et al. [62] combined the best-worst method (BWM) and the interval type 2 fuzzy TOPSIS methods to solve green supplier selection problem.

\section{Methodology}

Maniya and Bhatt [63] introduced preference selection index (PSI) to solve material selection problem. The range of value (ROV) was developed by Yakowitz et al. [64]. The ROV method is one of the scoring methods. The easiest MADM methods are scoring methods [65]. However, there are limited studies related to ROV method in the literature. While most of these studies were using the crisp ROV method, Zavadskas et al. [66] developed the rough ROV method. In this study, FPSI and FROV are used to select the best supplier by considering environmental aspects. FPSI is used to identify the objective weights of attributes and FROV is used to order the suppliers with respect to their performances. Methodology section consists of three subsections including fuzzy arithmetic operations, FPSI, and FROV methods.

3.1. Fuzzy Arithmetic Operations. It can be supposed that the arithmetic operations are used for the fuzzy numbers and crisp numbers. $\widetilde{G}=\left(g^{l}, g^{m}, g^{u}\right)$ and $\widetilde{H}=\left(h^{l}, h^{m}, h^{u}\right)$ represent the two positive triangular fuzzy numbers. More details are indicated as follows [67].

(i) Addition: $\widetilde{G}+\widetilde{H}=\left(g^{l}+h^{l}, g^{m}+h^{m}, g^{u}+h^{u}\right)$

(ii) Subtraction: $\widetilde{G}-\widetilde{H}=\left(g^{l}-h^{l}, g^{m}-h^{m}, g^{u}-h^{u}\right)$

(iii) Multiplication: $\widetilde{G} \times \widetilde{H}=\left(\min \left(g^{l} h^{l}, g^{l} h^{u}, g^{u} h^{l}, g^{u} h^{u}\right)\right.$, $\left.g^{m} h^{m}, \max \left(g^{l} h^{l}, g^{l} h^{u}, g^{u} h^{l}, g^{u} h^{u}\right)\right)$

(iv) Division: $\widetilde{G} / \widetilde{H}=\left(\min \left(g^{l} / h^{l}, g^{l} / h^{u}, g^{u} / h^{l}, g^{u} / h^{u}\right)\right.$, $\left.g^{m} / h^{m}, \max \left(g^{l} / h^{l}, g^{l} / h^{u}, g^{u} / h^{l}, g^{u} / h^{u}\right)\right)$

The e represents a positive crisp number [68].

(v) Scalar division: $\widetilde{G} / e=\left(g^{l} / e, g^{m} / e, g^{u} / e\right)$.

3.2. Fuzzy Preference Selection Index. FPSI method consists of five steps which are explained as follows.

Step 1-1. Decision-makers used Table 3 to assign linguistic performance rating. Fuzzy performance rating of decisionmakers is aggregated by using (1) to structure aggregated fuzzy decision matrix $(\widetilde{\mathrm{D}})$ :

$$
\tilde{d}_{i j}=\frac{1}{K} \sum_{k=1}^{K} \widetilde{d}_{i j k}
$$

In (1), $\tilde{d}_{i j k}$ denotes $k$ th decision-maker's fuzzy performance rating $\left(\widetilde{d}_{i j}\right)$ and $\widetilde{d}_{i j}$ is fuzzy performance rating of 
$i$ th alternative on $j$ th attribute and it is an element of $\widetilde{D}=$ $\left[\widetilde{d}_{i j}\right]_{m \times n}$.

Step 1-2. After structuring aggregated fuzzy decision matrix, the normalized fuzzy performance rating $\left(\widetilde{t}_{i j}\right)$, which is an element of normalized fuzzy decision matrix $\left(\widetilde{T}=\left[\widetilde{t}_{i j}\right]_{m \times n}\right)$, is calculated by using (2) (beneficial attributes) and (3) (nonbeneficial attributes) indicated as follows.

$$
\begin{aligned}
& \tilde{t}_{i j}=\frac{\widetilde{d}_{i j}}{\max \left(\widetilde{d}_{i j}\right)} \\
& \tilde{t}_{i j}=\frac{\min \left(\widetilde{d}_{i j}\right)}{\widetilde{d}_{i j}}
\end{aligned}
$$

Step 1-3. The averaged fuzzy normalized value $\left(\overline{\vec{t}}_{i j}\right)$ of each attribute is computed by

$$
\overline{\tilde{t}}_{i j}=\frac{1}{m} \sum_{i=1}^{m} \widetilde{t}_{i j}
$$

Step 1-4. The fuzzy preference value $\left(\widetilde{P V}_{j}=\right.$ $\left.\left(P V_{j}^{l}, P V_{j}^{m}, P V_{j}^{u}\right)\right)$ of each attribute is calculated as follows.

$$
\widetilde{P V}_{j}=\sum_{i=1}^{m}\left(\widetilde{t}_{i j}-\overline{\tilde{t}}_{i j}\right)^{2}
$$

Step 1-5. The fuzzy deviation value $\left(\widetilde{\sigma}_{j}\right)$ of each attribute is computed by (6). Then, fuzzy weight $\left(\widetilde{w}_{j}\right)$ of each attribute is calculated by (7) and fuzzy normalized weight $\left(\widetilde{w}_{j}^{*}\right)$ is computed by (8).

$$
\begin{aligned}
\widetilde{\sigma}_{j} & =\left(\sigma_{j}^{l}, \sigma_{j}^{m}, \sigma_{j}^{u}\right)=\left|1-\widetilde{P V}_{j}\right| \\
& =\left(\left|1-P V_{j}^{u}\right|,\left|1-P V_{j}^{m}\right|,\left|1-P V_{j}^{l}\right|\right) \\
\widetilde{w}_{j} & =\frac{\widetilde{\sigma}_{j}}{\sum_{j=1}^{n} \widetilde{\sigma}_{j}} \\
\widetilde{w}_{j}^{*} & =\frac{3 \times \widetilde{w}_{j}}{\sum_{j=1}^{n} w_{j}^{l}+\sum_{j=1}^{n} w_{j}^{m}+\sum_{j=1}^{n} w_{j}^{u}}
\end{aligned}
$$

After obtaining the fuzzy normalized weight of each attribute, these weights are transferred into FROV method.

\subsection{Fuzzy Range of Value. FROV method contains four steps} indicated as follows.

Step 2-1. The range of fuzzy values placed in the aggregated fuzzy decision matrix $(\widetilde{D})$, which is structured in (1), is obtained by (9) (beneficial attributes) and (10) (nonbeneficial attributes).

$$
\begin{aligned}
\widetilde{s}_{i j} & =\frac{\widetilde{d}_{i j}-\min \left(\widetilde{d}_{i j}\right)}{\max \left(\widetilde{d}_{i j}\right)-\min \left(\widetilde{d}_{i j}\right)} \\
& =\left(\frac{d_{i j}^{l}-\min \left(d_{i j}^{u}\right)}{\max \left(d_{i j}^{l}\right)-\min \left(d_{i j}^{l}\right)},\right. \\
& \left.\frac{d_{i j}^{m}-\min \left(d_{i j}^{m}\right)}{\max \left(d_{i j}^{m}\right)-\min \left(d_{i j}^{m}\right)}, \frac{d_{i j}^{u}-\min \left(d_{i j}^{l}\right)}{\max \left(d_{i j}^{u}\right)-\min \left(d_{i j}^{l}\right)}\right) \\
\widetilde{s}_{i j} & =\frac{\max \left(\widetilde{d}_{i j}\right)-\widetilde{d}_{i j}}{\max \left(\widetilde{d}_{i j}\right)-\min \left(\widetilde{d}_{i j}\right)} \\
& =\left(\frac{\max \left(d_{i j}^{l}\right)-d_{i j}^{u}}{\max \left(d_{i j}^{l}\right)-\min \left(d_{i j}^{l}\right)},\right. \\
& \left.\frac{\max \left(d_{i j}^{m}\right)-d_{i j}^{m}}{\max \left(d_{i j}^{m}\right)-\min \left(d_{i j}^{m}\right)}, \frac{\max \left(d_{i j}^{u}\right)-d_{i j}^{l}}{\max \left(d_{i j}^{u}\right)-\min \left(d_{i j}^{l}\right)}\right)
\end{aligned}
$$

In (9) and (10), $\widetilde{s}_{i j}$, which is an element of the fuzzy range decision matrix $\left(\widetilde{S}=\left[\widetilde{s}_{i j}\right]_{m \times n}\right)$, indicates the range of fuzzy values.

Step 2-2. After this, fuzzy worst utility values $\left(\widetilde{\mathcal{u}}_{i}^{-}\right)$and fuzzy best utility values $\left(\widetilde{u}_{i}^{+}\right)$for each alternative are computed as follows.

$$
\begin{aligned}
& \widetilde{u}_{i}^{-}=\sum_{i=1}^{f} \widetilde{s}_{i j} \widetilde{w}_{j} \\
& \widetilde{u}_{i}^{+}=\sum_{i=f+1}^{m} \widetilde{s}_{i j} \widetilde{w}_{j}
\end{aligned}
$$

Step 2-3. Fuzzy overall score $\left(\widetilde{u}_{i}=\left(u_{i}^{l}, u_{i}^{m}, u_{i}^{u}\right)\right)$ for each alternative is calculated by

$$
\widetilde{u}_{i}=\frac{\widetilde{u}_{i}^{-}+\tilde{u}_{i}^{+}}{2}
$$

Step 2-4. Fuzzy overall scores $\left(\widetilde{u}_{i}\right)$ are converted into crisp overall score $\left(u_{i}\right)$ by using

$$
u_{i}=\frac{u_{i}^{l}+u_{i}^{m}+u_{i}^{u}}{3}
$$

Then, alternatives are ordered from the highest crisp overall score to the lowest crisp overall score. The alternative having the highest crisp overall score is identified as the most appropriate alternative.

\section{Application}

The hybrid fuzzy model is applied into a textile company, which has more than 10 years of experience in the 
TABLE 4: The aggregated fuzzy decision matrix.

\begin{tabular}{|c|c|c|c|}
\hline \multirow{2}{*}{ Suppliers } & \multicolumn{3}{|c|}{ Attributes } \\
\hline & $\mathrm{A} 1$ & $\mathrm{~A} 2$ & $\mathrm{~A} 3$ \\
\hline Supplier 1 & $(2.5,3,3.2)$ & $(0.020,0.022,0.024)$ & $(0.012,0.013,0.014)$ \\
\hline Supplier 2 & $(3.2,3.3,3.4)$ & $(0.017,0.021,0.023)$ & $(0.011,0.012,0.015)$ \\
\hline Supplier 3 & $(2.8,3,3.1)$ & $(0.019,0.024,0.025)$ & $(0.009,0.010,0.017)$ \\
\hline Supplier 4 & $(2.9,3.1,3.4)$ & $(0.018,0.023,0.024)$ & $(0.010,0.011,0.012)$ \\
\hline Supplier 5 & $(3.2,3.3,3.5)$ & $(0.016,0.019,0.021)$ & $(0.008,0.010,0.015)$ \\
\hline Supplier 6 & $(3.1,3.2,3.4)$ & $(0.018,0.021,0.022)$ & $(0.010,0.014,0.016)$ \\
\hline Supplier 7 & $(3.1,3.3,3.5)$ & $(0.019,0.022,0.023)$ & $(0.010,0.011,0.013)$ \\
\hline Supplier 8 & $(3.2,3.4,3.5)$ & $(0.017,0.021,0.024)$ & $(0.010,0.012,0.014)$ \\
\hline \multirow{2}{*}{ Suppliers } & \multicolumn{3}{|c|}{ Attributes } \\
\hline & A4 & A5 & A6 \\
\hline Supplier 1 & $(3.8,5.8,7.8)$ & $(4.6,6.6,8.6)$ & $(3,5,7)$ \\
\hline Supplier 2 & $(3,5,7)$ & $(4.2,6.2,8.2)$ & $(3.4,5.4,7.4)$ \\
\hline Supplier 3 & $(4.2,6.2,8.2)$ & $(4.6,6.6,8.6)$ & $(3.4,5.4,7.4)$ \\
\hline Supplier 4 & $(4.6,6.6,8.6)$ & $(3,5,7)$ & $(3.8,5.8,7.8)$ \\
\hline Supplier 5 & $(5,7,9)$ & $(3.4,5.4,7.4)$ & $(5,7,9)$ \\
\hline Supplier 6 & $(3.8,5.8,7.8)$ & $(7,9,10)$ & $(3,5,7)$ \\
\hline Supplier 7 & $(5,7,9)$ & $(4.6,6.6,8.6)$ & $(3,5,7)$ \\
\hline Supplier 8 & $(7,9,10)$ & $(5.4,7.4,9.2)$ & $(2.2,4.2,6.2)$ \\
\hline \multirow{2}{*}{ Suppliers } & \multicolumn{3}{|c|}{ Attributes } \\
\hline & A7 & A8 & A9 \\
\hline Supplier 1 & $(2.2,4.2,6.2)$ & $(1,3,5)$ & $(1.8,3.8,5.8)$ \\
\hline Supplier 2 & $(1,3,5)$ & $(0,1,3)$ & $(0,1,3)$ \\
\hline Supplier 3 & $(2.2,4.2,6.2)$ & $(1.4,3.4,5.4)$ & $(1.4,3.4,5.4)$ \\
\hline Supplier 4 & $(3,5,7)$ & $(2.6,4.6,6.6)$ & $(1,3,5)$ \\
\hline Supplier 5 & $(4.6,6.6,8.6)$ & $(3,5,7)$ & $(2.2,4.2,6.2)$ \\
\hline Supplier 6 & $(2.2,4.2,6.2)$ & $(1.8,3.8,5.8)$ & $(1.8,3.8,5.8)$ \\
\hline Supplier 7 & $(1,3,5)$ & $(2.2,4.2,6.2)$ & $(1,3,5)$ \\
\hline Supplier 8 & $(1,3,5)$ & $(1.4,3.4,5.4)$ & $(1,3,5)$ \\
\hline
\end{tabular}

sector manufacturing suits for global market. The buyers of the suits motivate the company to work with green suppliers. Before interviewing with managers of company, attribute list was structured by means of literature. Then, the company managers were asked whether the attributes were appropriate for the company in the supplier selection process. Nine attributes were identified for using in supplier selection. These attributes are Cost (A1), Defective Rate (A2), Late Delivery Rate (A3), Technological Capability (A4), Technical Assistance (A5), Pollution Control (A6), Environmental Management (A7), Green Transportation (A8), and Green Warehousing (A9). The first three attributes are identified as nonbeneficial attributes and the others are identified as beneficial attributes. This company procures yarn (thread spools) from 8 suppliers. The fuzzy data of the first three attributes were obtained from factory manager considering actual data of company. The fuzzy data of other attributes were collected from five managers of company including factory manager, purchasing manager, planning manager, operation manager, and quality manager. The aggregated fuzzy decision matrix is indicated in Table 4.
By using (2) and (3), the aggregated fuzzy decision matrix is normalized. The normalized fuzzy decision matrix is demonstrated in Table 5.

By means of (5), the fuzzy preference value $\left(\widetilde{P V}_{j}\right)$ of each attribute is computed. After obtaining $\widetilde{P V}_{\mathrm{j}}$, the fuzzy deviation value $\left(\widetilde{\sigma}_{\mathrm{j}}\right)$ of each attribute is calculated by using $(6)$. Then, fuzzy weight $\left(\widetilde{w}_{j}\right)$ and fuzzy normalized weight $\left(\widetilde{w}_{j}^{*}\right)$ of each attribute is computed by using (7) and (8), respectively. These results are indicated in Table 6.

The fuzzy weights of attributes are considered into FROV. By means of (9) and (10), the fuzzy range decision matrix $(\widetilde{S})$, which is indicated in Table 7 , is calculated.

In final step, the fuzzy best and worst utility values $\left(\widetilde{u}_{i}^{+}, \widetilde{u}_{i}^{-}\right)$of each supplier are calculated by using (11) and (12), respectively. These values are aggregated by (13) to obtain fuzzy overall score $\left(\widetilde{u}_{i}\right)$ for each alternative and these fuzzy scores are converted into crisp overall score $\left(u_{i}\right)$ by using $(14)$. These results are indicated in Table 8 .

According to crisp overall score $\left(u_{i}\right)$ indicated in Table 8 , the ranking of suppliers are as follows: Supplier 5, Supplier 4, Supplier 6, Supplier 3, Supplier 1, Supplier 8, and Supplier 2. 
TABLE 5: The normalized fuzzy decision matrix (for FPSI).

\begin{tabular}{|c|c|c|c|}
\hline \multirow{2}{*}{ Suppliers } & \multicolumn{3}{|c|}{ Attributes } \\
\hline & $\mathrm{A} 1$ & $\mathrm{~A} 2$ & A3 \\
\hline Supplier 1 & $(0.781,1,1.24)$ & $(0.667,0.864,1.050)$ & $(0.571,0.769,1)$ \\
\hline Supplier 2 & $(0.735,0.909,0.969)$ & $(0.696,0.905,1.235)$ & $(0.533,0.833,1.091)$ \\
\hline Supplier 3 & $(0.806,1,1.107)$ & $(0.640,0.792,1.105)$ & $(0.471,1,1.333)$ \\
\hline Supplier 4 & $(0.735,0.968,1.069)$ & $(0.667,0.826,1.167)$ & $(0.667,0.909,1.2)$ \\
\hline Supplier 5 & $(0.714,0.909,0.969)$ & $(0.762,1,1.313)$ & $(0.533,1,1.5)$ \\
\hline Supplier 6 & $(0.735,0.938,1)$ & $(0.727,0.905,1.167)$ & $(0.5,0.714,1.2)$ \\
\hline Supplier 7 & $(0.714,0.909,1)$ & $(0.696,0.864,1.105)$ & $(0.615,0.909,1.2)$ \\
\hline Supplier 8 & $(0.714,0.882,0.969)$ & $(0.667,0.905,1.235)$ & $(0.571,0.833,1.2)$ \\
\hline \multirow{2}{*}{ Suppliers } & \multicolumn{3}{|c|}{ Attributes } \\
\hline & A4 & A5 & A6 \\
\hline Supplier 1 & $(0.380,0.644,1.114)$ & $(0.460,0.733,1.229)$ & $(0.333,0.714,1.4)$ \\
\hline Supplier 2 & $(0.3,0.556,1)$ & $(0.420,0.689,1.171)$ & $(0.378,0.771,1.48)$ \\
\hline Supplier 3 & $(0.420,0.689,1.171)$ & $(0.460,0.733,1.229)$ & $(0.378,0.771,1.48)$ \\
\hline Supplier 4 & $(0.460,0.733,1.229)$ & $(0.3,0.556,1)$ & $(0.422,0.829,1.56)$ \\
\hline Supplier 5 & $(0.5,0.778,1.286)$ & $(0.340,0.6,1.057)$ & $(0.556,1,1.8)$ \\
\hline Supplier 6 & $(0.380,0.644,1.114)$ & $(0.7,1,1.429)$ & $(0.333,0.714,1.4)$ \\
\hline Supplier 7 & $(0.5,0.778,1.286)$ & $(0.460,0.733,1.229)$ & $(0.333,0.714,1.4)$ \\
\hline Supplier 8 & $(0.7,1,1.429)$ & $(0.540,0.822,1.314)$ & $(0.244,0.6,1.240)$ \\
\hline \multirow{2}{*}{ Suppliers } & \multicolumn{3}{|c|}{ Attributes } \\
\hline & A7 & A8 & A9 \\
\hline Supplier 1 & $(0.256,0.636,1.348)$ & $(0.143,0.6,1.667)$ & $(0.290,0.905,2.636)$ \\
\hline Supplier 2 & $(0.116,0.455,1.087)$ & $(0,0.2,1)$ & $(0,0.238,1.364)$ \\
\hline Supplier 3 & $(0.256,0.636,1.348)$ & $(0.2,0.680,1.8)$ & $(0.226,0.810,2.455)$ \\
\hline Supplier 4 & $(0.349,0.758,1.522)$ & $(0.371,0.920,2.2)$ & $(0.161,0.714,2.273)$ \\
\hline Supplier 5 & $(0.535,1,1.870)$ & $(0.429,1,2.333)$ & $(0.355,1,2.818)$ \\
\hline Supplier 6 & $(0.256,0.636,1.348)$ & $(0.257,0.760,1.933)$ & $(0.290,0.905,2.636)$ \\
\hline Supplier 7 & $(0.116,0.455,1.087)$ & $(0.314,0.840,2.067)$ & $(0.161,0.714,2.273)$ \\
\hline Supplier 8 & $(0.116,0.455,1.087)$ & $(0.2,0.680,1.8)$ & $(0.161,0.714,2.273)$ \\
\hline
\end{tabular}

TABLE 6: The results of FPSI.

\begin{tabular}{|c|c|c|c|}
\hline \multirow{2}{*}{ Results } & \multicolumn{3}{|c|}{ Attributes } \\
\hline & $\mathrm{A} 1$ & $\mathrm{~A} 2$ & $\mathrm{~A} 3$ \\
\hline${\widetilde{\mathrm{PV}_{j}}}$ & $(0.009,0.015,0.064)$ & $(0.012,0.025,0.051)$ & $(0.028,0.073,0.158)$ \\
\hline$\widetilde{\sigma}_{j}$ & $(0.936,0.985,0.991)$ & $(0.949,0.975,0.988)$ & $(0.842,0.927,0.972)$ \\
\hline$\widetilde{\mathrm{w}}_{\mathrm{j}}$ & $(0.112,0.132,0.156)$ & $(0.114,0.130,0.156)$ & $(0.101,0.124,0.153)$ \\
\hline$\widetilde{w}_{j}^{*}$ & $(0.109,0.129,0.152)$ & $(0.111,0.127,0.152)$ & $(0.099,0.121,0.149)$ \\
\hline \multirow{2}{*}{ Results } & \multicolumn{3}{|c|}{ Attributes } \\
\hline & $\mathrm{A} 4$ & A5 & A6 \\
\hline$\overline{\widetilde{P V}}_{j}$ & $(0.101,0.125,0.126)$ & $(0.106,0.127,0.130)$ & $(0.059,0.096,0.185)$ \\
\hline$\widetilde{\sigma}_{j}$ & $(0.874,0.875,0.899)$ & $(0.870,0.873,0.894)$ & $(0.815,0.904,0.941)$ \\
\hline$\widetilde{\mathrm{w}}_{j}$ & $(0.105,0.117,0.142)$ & $(0.105,0.116,0.141)$ & $(0.098,0.121,0.148)$ \\
\hline$\widetilde{w}_{j}^{*}$ & $(0.102,0.114,0.139)$ & $(0.102,0.113,0.138)$ & $(0.096,0.118,0.144)$ \\
\hline \multirow{2}{*}{ Results } & \multicolumn{3}{|c|}{ Attributes } \\
\hline & A7 & A8 & A9 \\
\hline${\widetilde{\mathrm{PV}_{j}}}$ & $(0.145,0.245,0.507)$ & $(0.129,0.422,1.172)$ & $(0.084,0.380,1.385)$ \\
\hline$\tilde{\sigma}_{j}$ & $(0.493,0.755,0.855)$ & $(0.172,0.578,0.871)$ & $(0.385,0.620,0.916)$ \\
\hline$\widetilde{\mathrm{w}}_{\mathrm{j}}$ & $(0.059,0.101,0.135)$ & $(0.021,0.077,0.137)$ & $(0.046,0.083,0.144)$ \\
\hline$\widetilde{w}_{j}^{*}$ & $(0.058,0.099,0.132)$ & $(0.020,0.075,0.134)$ & $(0.045,0.081,0.141)$ \\
\hline
\end{tabular}


TABLE 7: The fuzzy range decision matrix.

\begin{tabular}{|c|c|c|c|}
\hline \multirow{2}{*}{ Suppliers } & \multicolumn{3}{|c|}{ Attributes } \\
\hline & $\mathrm{A} 1$ & $\mathrm{~A} 2$ & A3 \\
\hline Supplier 1 & $(0,1,1.429)$ & $(-1,0.400,1.250)$ & $(-0.500,0.250,1.250)$ \\
\hline Supplier 2 & $(-0.286,0.250,0.429)$ & $(-0.750,0.600,2)$ & $(-0.750,0.500,1.500)$ \\
\hline Supplier 3 & $(0.100,1,1)$ & $(-1.250,0,1.500)$ & $(-1.250,1,2)$ \\
\hline Supplier 4 & $(-0.286,0.750,0.857)$ & $(-1,0.200,1.750)$ & $(0,0.750,1.750)$ \\
\hline Supplier 5 & $(-0.429,0.250,0.429)$ & $(-0.250,1,2.250)$ & $(-0.750,1,2.250)$ \\
\hline Supplier 6 & $(-0.286,0.500,0.571)$ & $(-0.500,0.600,1.750)$ & $(-1,0,1.750)$ \\
\hline Supplier 7 & $(-0.429,0.250,0.571)$ & $(-0.750,0.400,1.500)$ & $(-0.250,0.750,1.750)$ \\
\hline Supplier 8 & $(-0.429,0,0.429)$ & $(-1,0.600,2)$ & $(-0.500,0.500,1.750)$ \\
\hline \multirow{2}{*}{ Suppliers } & \multicolumn{3}{|c|}{ Attributes } \\
\hline & A4 & A5 & A6 \\
\hline Supplier 1 & $(-0.800,0.200,1.200)$ & $(-0.600,0.400,1.400)$ & $(-1.143,0.286,1.714)$ \\
\hline Supplier 2 & $(-1,0,1)$ & $(-0.700,0.300,1.300)$ & $(-1,0.429,1.857)$ \\
\hline Supplier 3 & $(-0.700,0.300,1.300)$ & $(-0.600,0.400,1.400)$ & $(-1,0.429,1.857)$ \\
\hline Supplier 4 & $(-0.600,0.400,1.400)$ & $(-1,0,1)$ & $(-0.857,0.571,2)$ \\
\hline Supplier 5 & $(-0.500,0.500,1.500)$ & $(-0.900,0.100,1.100)$ & $(-0.429,1,2.429)$ \\
\hline Supplier 6 & $(-0.800,0.200,1.200)$ & $(0,1,1.750)$ & $(-1.143,0.286,1.714)$ \\
\hline Supplier 7 & $(-0.500,0.500,1.500)$ & $(-0.600,0.400,1.400)$ & $(-1.143,0.286,1.714)$ \\
\hline Supplier 8 & $(0,1,1.750)$ & $(-0.400,0.600,1.550)$ & $(-1.429,0,1.429)$ \\
\hline \multirow{2}{*}{ Suppliers } & \multicolumn{3}{|c|}{ Attributes } \\
\hline & A7 & A8 & A9 \\
\hline Supplier 1 & $(-0.778,0.333,1.444)$ & $(-0.667,0.500,1.667)$ & $(-0.546,0.875,2.636)$ \\
\hline Supplier 2 & $(-1.111,0,1.111)$ & $(-1,0,1)$ & $(-1.364,0,1.364)$ \\
\hline Supplier 3 & $(-0.778,0.333,1.444)$ & $(-0.533,0.600,1.800)$ & $(-0.727,0.750,2.455)$ \\
\hline Supplier 4 & $(-0.556,0.556,1.667)$ & $(-0.133,0.900,2.200)$ & $(-0.909,0.625,2.273)$ \\
\hline Supplier 5 & $(-0.111,1,2.111)$ & $(0,1,2.333)$ & $(-0.364,1,2.818)$ \\
\hline Supplier 6 & $(-0.778,0.333,1.444)$ & $(-0.400,0.700,1.933)$ & $(-0.546,0.875,2.636)$ \\
\hline Supplier 7 & $(-1.111,0,1.111)$ & $(-0.267,0.800,2.067)$ & $(-0.909,0.625,2.273)$ \\
\hline Supplier 8 & $(-1.111,0,1.111)$ & $(-0.533,0.600,1.800)$ & $(-0.909,0.625,2.273)$ \\
\hline
\end{tabular}

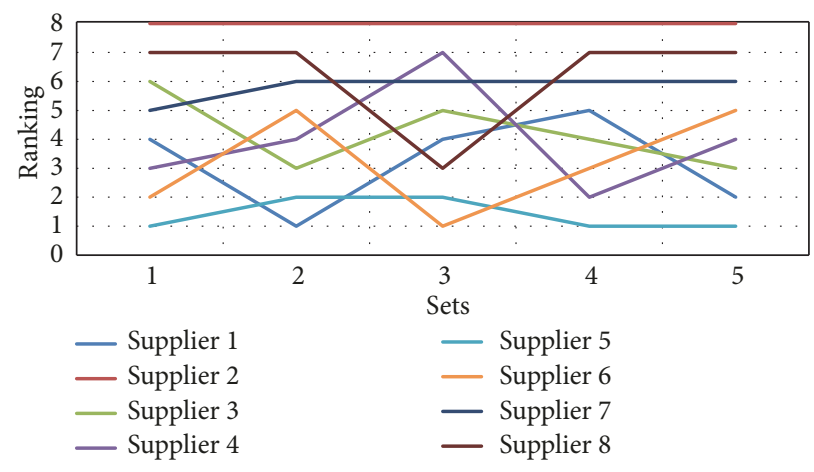

FIGURE 1: The results of sensitivity analysis.

Therefore, the best supplier among 8 suppliers is identified as Supplier 5.

The results of FROV are compared with the results of other fuzzy MADM, which are fuzzy ARAS, fuzzy MULTIMOORA, fuzzy COPRAS, and fuzzy GRA. Table 9 presents the coefficient of Spearman's correlation for all other fuzzy MADM.

According to Table 9, the correlation between the results of FROV and the results of other fuzzy MADM methods is very high. Table 9 proves that the FROV method has reached 
TABLE 8: The results of FROV.

\begin{tabular}{lcr}
\hline Suppliers & \multicolumn{1}{c}{ Results } & $\widetilde{u}_{i}^{+}$ \\
\hline Supplier 1 & $(-0.227,0.210,0.593)$ & $(-0.628,0.244,1.393)$ \\
Supplier 2 & $(-0.269,0.169,0.593)$ & $(-0.853,0.085,1.058)$ \\
Supplier 3 & $(-0.365,0.250,0.678)$ & $(-0.601,0.269,1.419)$ \\
Supplier 4 & $(-0.195,0.213,0.657)$ & $(-0.563,0.287,1.456)$ \\
Supplier 5 & $(-0.215,0.280,0.742)$ & $(-0.322,0.441,1.700)$ \\
Supplier 6 & $(-0.268,0.141,0.614)$ & $(-0.510,0.327,1.478)$ \\
Supplier 7 & $(-0.216,0.174,0.576)$ & $(-0.629,0.247,1.393)$ \\
Supplier 8 & $(-0.292,0.137,0.630)$ & $(-0.607,0.278,1.371)$ \\
\hline Suppliers & & Results \\
Supplier 1 & $\widetilde{u}_{i}$ & 0.264 \\
Supplier 2 & $(-0.428,0.227,0.993)$ & 0.131 \\
Supplier 3 & $(-0.561,0.127,0.826)$ & 0.275 \\
Supplier 4 & $(-0.483,0.260,1.049)$ & 0.309 \\
Supplier 5 & $(-0.379,0.250,1.057)$ & 0.438 \\
Supplier 6 & $(-0.269,0.361,1.221)$ & 0.297 \\
Supplier 7 & $(-0.389,0.234,1.046)$ & 0.258 \\
Supplier 8 & $(-0.423,0.211,0.985)$ & 0.253 \\
\hline
\end{tabular}

TABLE 9: Spearman correlation coefficient for all fuzzy MADM.

\begin{tabular}{lccccc}
\hline Fuzzy MADM & FROV & Fuzzy ARAS & $\begin{array}{c}\text { Fuzzy } \\
\text { MULTIMOORA }\end{array}$ & Fuzzy COPRAS & Fuzzy GRA \\
\hline FROV & 1.000 & 0.952 & 0.929 & 0.881 & 0.833 \\
Fuzzy ARAS & - & 1.000 & 0.916 & 0.952 & 0.952 \\
Fuzzy MULTIMOORA & - & - & 1.000 & 0.952 & 0.857 \\
Fuzzy COPRAS & - & - & - & -0.952 & 1.000 \\
Fuzzy GRA & - & - & - & & - \\
\hline
\end{tabular}

the accurate results. Additionally, FROV method includes few and simple steps. It can easily be used to solve MADM problems.

\section{Sensitivity Analysis}

The sensitivity analysis is done to monitor the changing of the results with respect to the changing of attribute weights. For this purpose, five sets of attribute weights are determined. Table 10 presents the sets of attribute weights.

These attribute weights are used to do the sensitivity analysis. The results of the sensitivity analysis are presented in Figure 1.

As it can be seen that Supplier 5 is determined as the best supplier for Sets 1, 4, and 5, nevertheless, Supplier 1 is identified as the best supplier in Set 2 and Supplier 6 is determined as the best supplier in Set 3. Only one supplier's rank does not change. That supplier is Supplier 2 and this supplier is always 8 th rank. The ranking of other suppliers varies at least once with respect to the sets of attribute weights.

\section{Conclusion}

This study's main objective was to develop a hybrid model to choose suppliers in accordance with sustainability and for this purpose it made three contributions to green supplier selection literature. First contribution is proposing a new method which is FROV to literature, second contribution is utilizing FPSI to identify the weights of attributes, and third contribution is developing a new MADM model consisting of FPSI and FROV to solve supplier selection.

Choosing the most suitable attributes and the method to be used in the decision model is significant for green supplier selection. Therefore, first, a review of attributes used in the selection was conducted and a comprehensive list of suitable attributes for selecting green suppliers was created. Then, an interview was held with the managers of a textile company to shape a final list of applicable attributes for this study. Nine attributes were identified for using in supplier selection. These attributes are Cost (A1), Defective Rate (A2), Late Delivery Rate (A3), Technological Capability (A4), Technical 
TABLE 10: Sensitivity analysis.

\begin{tabular}{|c|c|c|c|}
\hline \multirow{2}{*}{ Attributes } & \multicolumn{3}{|c|}{ Sets } \\
\hline & Set 1 & Set 2 & Set 3 \\
\hline Attribute 1 & $(0.036,0.043,0.051)$ & $(0.370,0.390,0.450)$ & $(0.200,0.240,0.450)$ \\
\hline Attribute 2 & $(0.037,0.042,0.051)$ & $(0.012,0.014,0.017)$ & $(0.150,0.180,0.190)$ \\
\hline Attribute 3 & $(0.033,0.040,0.050)$ & $(0.011,0.013,0.017)$ & $(0.020,0.050,0.070)$ \\
\hline Attribute 4 & $(0.051,0.057,0.070)$ & $(0.015,0.019,0.023)$ & $(0.210,0.220,0.230)$ \\
\hline Attribute 5 & $(0.051,0.057,0.069)$ & $(0.017,0.019,0.023)$ & $(0.200,0.220,0.260)$ \\
\hline Attribute 6 & $(0.048,0.059,0.070)$ & $(0.060,0.080,0.090)$ & $(0.010,0.020,0.030)$ \\
\hline Attribute 7 & $(0.029,0.050,0.066)$ & $(0.040,0.060,0.070)$ & $(0.020,0.030,0.040)$ \\
\hline Attribute 8 & $(0.300,0.320,0.330)$ & $(0.140,0.200,0.240)$ & $(0.010,0.020,0.040)$ \\
\hline Attribute 9 & $(0.290,0.330,0.370)$ & $(0.140,0.190,0.280)$ & $(0.020,0.030,0.040)$ \\
\hline \multirow{2}{*}{ Attributes } & \multicolumn{3}{|c|}{ Sets } \\
\hline & Set 4 & \multicolumn{2}{|c|}{ Set 5} \\
\hline Attribute 1 & $(0.010,0.030,0.070)$ & \multicolumn{2}{|c|}{$(0.300,0.320,0.350)$} \\
\hline Attribute 2 & $(0.020,0.030,0.040)$ & \multicolumn{2}{|c|}{$(0.160,0.190,0.200)$} \\
\hline Attribute 3 & $(0.050,0.060,0.080)$ & \multicolumn{2}{|c|}{$(0.160,0.180,0.190)$} \\
\hline Attribute 4 & $(0.070,0.080,0.090)$ & \multicolumn{2}{|c|}{$(0.050,0.070,0.090)$} \\
\hline Attribute 5 & $(0.010,0.020,0.040)$ & \multicolumn{2}{|c|}{$(0.080,0.140,0.150)$} \\
\hline Attribute 6 & $(0.120,0.140,0.160)$ & \multicolumn{2}{|c|}{$(0.010,0.020,0.040)$} \\
\hline Attribute 7 & $(0.150,0.170,0.190)$ & \multicolumn{2}{|c|}{$(0.020,0.030,0.040)$} \\
\hline Attribute 8 & $(0.200,0.220,0.230)$ & \multicolumn{2}{|c|}{$(0.020,0.030,0.040)$} \\
\hline Attribute 9 & $(0.220,0.249,0.251)$ & \multicolumn{2}{|c|}{$(0.020,0.040,0.060)$} \\
\hline
\end{tabular}

Assistance (A5), Pollution Control (A6), Environmental Management (A7), Green Transportation (A8), and Green Warehousing (A9).

In addition, this study provided a novel hybrid MADM model to select green supplier. The proposed model incorporated FPSI which is used to identify the weights of attributes and FROV which is used to order the suppliers with respect to their performances.

Future studies may use this model to solve other MADM problems, such as logistics provider selection, energy sources selection, and warehouse location selection.

\section{Data Availability}

All data used to support the findings of this study are included within the article.

\section{Conflicts of Interest}

The authors declare that they have no conflicts of interest.

\section{References}

[1] WCED, Our Common Future: Report of the World Commission on Environment and Development, 1987.

[2] F. Afzal, B. Lim, and D. Prasad, "An investigation of corporate approaches to sustainability in the construction industry," Procedia Engineering, vol. 180, pp. 202-210, 2017.

[3] R.-D. Chang, J. Zuo, Z.-Y. Zhao et al., "Sustainability attitude and performance of construction enterprises: a china study," Journal of Cleaner Production, vol. 172, pp. 1440-1451, 2018.
[4] R.-H. Chen, Y. Lin, and M.-L. Tseng, "Multiattributes analysis of sustainable development indicators in the construction minerals industry in China," Resources Policy, vol. 46, pp. 123$133,2015$.

[5] S. Safinia, Z. Al-Hinai, H. A. Yahia, and M. F. Abushammala, "Sustainable construction in sultanate of oman: factors effecting materials utilization," Procedia Engineering, vol. 196, pp. 980987, 2017.

[6] N. B. Dang, S. Momtaz, K. Zimmerman, P. Thi, and H. Nhung, "Effectiveness of formal institutions in managing marine fisheries for sustainable fisheries development: A case study of a coastal commune in Vietnam," Ocean Coastal Management, vol. 137, pp. 175-184, 2017.

[7] A. Fleming, R. M. Wise, H. Hansen, and L. Sams, "The sustainable development goals: A case study," Marine Policy, vol. 86, pp. 94-103, 2017.

[8] S. Hernández Aguado, I. Segado Segado, and T. J. Pitcher, "Towards sustainable fisheries: A multi-criteria participatory approach to assessing indicators of sustainable fishing communities: A case study from Cartagena (Spain)," Marine Policy, vol. 65, pp. 97-106, 2016.

[9] I. B. M. Kosamu, "Conditions for sustainability of small-scale fisheries in developing countries," Fisheries Research, vol. 161, pp. 365-373, 2015.

[10] A. Lucchetti, S. E. A. Kholeif, H. H. Mahmoud, and E. Notti, "Towards sustainable fisheries management in emerging markets: An overview of properties, gaps and opportunities in Egypt," Marine Policy, vol. 72, pp. 1-10, 2016.

[11] M. Rossetto, I. Bitetto, M. T. Spedicato et al., "Multi-criteria decision-making for fisheries management: A case study of Mediterranean demersal fisheries," Marine Policy, vol. 53, pp. 83-93, 2015. 
[12] S. S. Erzurumlu and Y. O. Erzurumlu, "Sustainable mining development with community using design thinking and multicriteria decision analysis," Resources Policy, vol. 46, pp. 6-14, 2015.

[13] S. Kusi-Sarpong, C. Bai, J. Sarkis, and X. Wang, "Green supply chain practices evaluation in the mining industry using a joint rough sets and fuzzy TOPSIS methodology," Resources Policy, vol. 46, pp. 86-100, 2015.

[14] S. Luthra, D. Garg, and A. Haleem, "An analysis of interactions among critical success factors to implement green supply chain management towards sustainability: An Indian perspective," Resources Policy, vol. 46, pp. 37-50, 2015.

[15] B. S. Pimentel, E. S. Gonzalez, and G. N. O. Barbosa, "Decisionsupport models for sustainable mining networks: Fundamentals and challenges," Journal of Cleaner Production, vol. 112, pp. 2145-2157, 2016.

[16] L. Shen, K. Muduli, and A. Barve, "Developing a sustainable development framework in the context of mining industries: AHP approach," Resources Policy, vol. 46, pp. 15-26, 2015.

[17] R. Sivakumar, D. Kannan, and P. Murugesan, "Green vendor evaluation and selection using AHP and Taguchi loss functions in production outsourcing in mining industry," Resources Policy, vol. 46, pp. 64-75, 2015.

[18] M. Abbasi and F. Nilsson, "Developing environmentally sustainable logistics," Transportation Research Part D: Transport and Environment, vol. 46, pp. 273-283, 2016.

[19] V. de Almeida Guimarães and I. C. Leal Junior, "Performance assessment and evaluation method for passenger transportation: a step toward sustainability," Journal of Cleaner Production, vol. 142, pp. 297-307, 2017.

[20] Y. Huizhe, M. Lihua, and S. Fangfang, "Evaluation of sustainable development ability for logistics enterprises based on unascertained measure," Procedia Engineering, vol. 15, pp. 4757-4762, 2011.

[21] M. Jedliński, "The position of green logistics in sustainable development of a smart green city," Procedia - Social and Behavioral Sciences, vol. 151, pp. 102-111, 2014.

[22] A. S. Santos and S. K. Ribeiro, "The use of sustainability indicators in urban passenger transport during the decisionmaking process: The case of Rio de Janeiro, Brazil," Current Opinion in Environmental Sustainability, vol. 5, no. 2, pp. 251260, 2013.

[23] O. Seroka-Stolka, "The development of green logistics for implementation sustainable development strategy in companies," Procedia - Social and Behavioral Sciences, vol. 151, pp. 302309, 2014.

[24] S. Zailani, K. Govindan, M. Iranmanesh, M. R. Shaharudin, and Y. Sia Chong, "Green innovation adoption in automotive supply chain: The Malaysian case," Journal of Cleaner Production, vol. 108, pp. 1115-1122, 2015.

[25] S. H. Cheraghi, M. Dadashzadeh, and M. Subramanian, "Critical success factors for supplier selection: an update," Journal of Applied Business Research (JABR), vol. 20, no. 2, pp. 91-108, 2011.

[26] V. Baskaran, S. Nachiappan, and S. Rahman, "Indian textile suppliers' sustainability evaluation using the grey approach," International Journal of Production Economics, vol. 135, no. 2, pp. 647-658, 2012.

[27] P. M. Simpson, J. A. Siguaw, and S. C. White, "Measuring the performance of suppliers: an analysis of evaluation processes," Journal of Supply Chain Management, vol. 38, no. 1, pp. 29-41, 2002.
[28] W. Ho, X. Xu, and P. K. Dey, "Multi-criteria decision making approaches for supplier evaluation and selection: a literature review," European Journal of Operational Research, vol. 202, no. 1, pp. 16-24, 2010.

[29] G. Büyüközkan and G. Çifçi, "A novel hybrid MCDM approach based on fuzzy DEMATEL, fuzzy ANP and fuzzy TOPSIS to evaluate green suppliers," Expert Systems with Applications, vol. 39, no. 3, pp. 3000-3011, 2012.

[30] G. W. Dickson, "An analysis of vendor selection systems and decisions," Journal of Purchasing, vol. 2, no. 1, pp. 5-17, 1966.

[31] D. Kannan, A. B. L. D. S. Jabbour, C. José, and C. J. C. Jabbour, "Selecting green suppliers based on GSCM practices: using fuzzy TOPSIS applied to a Brazilian electronics company," European Journal of Operational Research, vol. 233, no. 2, pp. 432-447, 2014.

[32] A. H. I. Lee, H. Kang, C. F. Hsu, and H. Hung, "A green supplier selection model for high-tech industry," Expert Systems with Applications, vol. 36, no. 4, pp. 7917-7927, 2009.

[33] R. J. Kuo, Y. C. Wang, and F. C. Tien, "Integration of artificial neural network and MADA methods for green supplier selection," Journal of Cleaner Production, vol. 18, no. 12, pp. 1161-1170, 2010.

[34] S. Luthra, K. Govindan, D. Kannan, S. K. Mangla, and C. P. Garg, "An integrated framework for sustainable supplier selection and evaluation in supply chains," Journal of Cleaner Production, vol. 140, pp. 1686-1698, 2017.

[35] M. R. Galankashi, A. Chegeni, A. Soleimanynanadegany et al., "Prioritizing green supplier selection criteria using fuzzy analytical network process," Procedia CIRP, vol. 26, pp. 689694, 2015.

[36] K. Govindan, S. Rajendran, J. Sarkis, and P. Murugesan, "Multi criteria decision making approaches for green supplier evaluation and selection: a literature review," Journal of Cleaner Production, vol. 98, pp. 66-83, 2015.

[37] D. Kannan, K. Govindan, and S. Rajendran, "Fuzzy axiomatic design approach based green supplier selection: a case study from Singapore," Journal of Cleaner Production, vol. 96, pp. 194208, 2015.

[38] G. Noci, "Designing, green, vendor rating systems for the assessment of a suppliers environmental performance," European Journal of Purchasing Supply Management, vol. 3, no. 2, pp. 103-114, 1997.

[39] R. Handfield, S. V. Walton, R. Sroufe, and S. A. Melnyk, "Applying environmental criteria to supplier assessment: a study in the application of the Analytical Hierarchy Process," European Journal of Operational Research, vol. 141, no. 1, pp. 7087, 2002.

[40] P. Humphreys, R. McIvor, and F. Chan, "Using case-based reasoning to evaluate supplier environmental management performance," Expert Systems with Applications, vol. 25, no. 2, pp. 141-153, 2003.

[41] G. Büyüközkan and G. Çifçi, "A novel fuzzy multi-criteria decision framework for sustainable supplier selection with incomplete information," Computers in Industry, vol. 62, no. 2 , pp. 164-174, 2011.

[42] D. Kannan, R. Khodaverdi, L. Olfat, A. Jafarian, and A. Diabat, "Integrated fuzzy multi criteria decision making method and multi-objective programming approach for supplier selection and order allocation in a green supply chain," Journal of Cleaner Production, vol. 47, pp. 355-367, 2013.

[43] S. H. Hashemi, A. Karimi, and M. Tavana, "An integrated green supplier selection approach with analytic network process 
and improved Grey relational analysis," International Journal of Production Economics, vol. 159, pp. 178-191, 2015.

[44] R. Rostamzadeh, K. Govindan, A. Esmaeili, and M. Sabaghi, "Application of fuzzy VIKOR for evaluation of green supply chain management practices," Ecological Indicators, vol. 49, pp. 188-203, 2014.

[45] A. Awasthi and G. Kannan, "Green supplier development program selection using NGT and VIKOR under fuzzy environment," Computers \& Industrial Engineering, vol. 91, pp. 100-108, 2016.

[46] Ö Uygun and A. Dede, "Performance evaluation of green supply chain management using integrated fuzzy multi-attributes decision making techniques," Computers \& Industrial Engineering, vol. 102, pp. 502-511, 2016.

[47] Z. Guo, H. Liu, D. Zhang, and J. Yang, "Green supplier evaluation and selection in apparel manufacturing using a fuzzy multi-attributes decision-making approach," Sustainability, vol. 9, no. 4, pp. 1-13, 2017.

[48] K.-Q. Wang, H.-C. Liu, L. Liu, and J. Huang, "Green supplier evaluation and selection using cloud model theory and the QUALIFLEX method," Sustainability, vol. 9, no. 5, p. 688, 2017.

[49] F. Vahidi, S. A. Torabi, and M. J. Ramezankhani, "Sustainable supplier selection and order allocation under operational and disruption risks," Journal of Cleaner Production, vol. 174, pp. 1351-1365, 2018.

[50] F. Yu, Y. Yang, and D. Chang, "Carbon footprint based green supplier selection under dynamic environment," Journal of Cleaner Production, vol. 170, pp. 880-889, 2018.

[51] S. Vachon and R. D. Klassen, "Environmental management and manufacturing performance: The role of collaboration in the supply chain," International Journal of Production Economics, vol. 111, no. 2, pp. 299-315, 2008.

[52] Q. Zhu and J. Sarkis, "An inter-sectoral comparison of green supply chain management in China: drivers and practices," Journal of Cleaner Production, vol. 14, no. 5, pp. 472-486, 2006.

[53] S. K. Srivastava, "Green supply-chain management: a state-ofthe-art literature review," International Journal of Management Reviews, vol. 9, no. 1, pp. 53-80, 2007.

[54] M. E. González, G. Quesada, and C. A. M. Monge, "Determining the importance of the supplier selection process in manufacturing: a case study," International Journal of Physical Distribution ¿amp; Logistics Management, vol. 34, no. 6, pp. 492-504, 2004.

[55] A. Amindoust, S. Ahmed, A. Saghafinia, and A. Bahreininejad, "Sustainable supplier selection: a ranking model based on fuzzy inference system," Applied Soft Computing, vol. 12, no. 6, pp. 1668-1677, 2012.

[56] M. Zamani, A. Rabbani, A. Yazdani-Chamzini, and Z. Turskis, "An integrated model for extending brand based on fuzzy ARAS and ANP methods," Journal of Business Economics and Management, vol. 15, no. 3, pp. 403-423, 2014.

[57] A. Baležentis, T. Baležentis, and W. K. M. Brauers, "Personnel selection based on computing with words and fuzzy MULTIMOORA," Expert Systems with Applications, vol. 39, no. 9, pp. 7961-7967, 2012.

[58] M. Yazdani, A. Alidoosti, and E. K. Zavadskas, "Risk analysis of critical infrastructures using fuzzy copras," Economic ResearchEkonomska Istraživanja, vol. 24, no. 4, pp. 27-40, 2015.

[59] A. T. Gumus, A. Yesim Yayla, E. Çelik, and A. Yildiz, "A combined fuzzy-AHP and fuzzy-GRA methodology for hydrogen energy storage method selection in Turkey," Energies, vol. 6, no. 6, pp. 3017-3032, 2013.
[60] H. M. W. Chen, S. Y. Chou, Q. D. Luu, and T. H. K. Yu, "A fuzzy MCDM approach for green supplier selection from the economic and environmental aspects," Mathematical Problems in Engineering, vol. 2016, Article ID 8097386, 10 pages, 2016.

[61] Q. Pang, T. Yang, M. Li, and Y. Shen, "A fuzzy-grey multicriteria decision making approach for green supplier selection in lowcarbon supply chain," Mathematical Problems in Engineering, vol. 2017, Article ID 9653261, 9 pages, 2017.

[62] M. Yucesan, S. Mete, F. Serin, E. Celik, and M. Gul, "An integrated best-worst and interval type-2 fuzzy topsis methodology for green supplier selection," Mathematics, vol. 7, no. 2, p. 182, 2019.

[63] K. Maniya and M. G. A. Bhatt, "A selection of material using a novel type decision-making method: Preference selection index method," Materials \& Design, vol. 31, no. 4, pp. 1785-1789, 2010.

[64] D. S. Yakowitz, L. J. Lane, and F. Szidarovszky, "Multi-attribute decision making: dominance with respect to an importance order of the attributes," Applied Mathematics and Computation, vol. 54, no. 2-3, pp. 167-181, 1993.

[65] V. Penadés-Plà, T. García-Segura, J. Martí, and V. Yepes, "A review of multi-criteria decision-making methods applied to the sustainable bridge design," Sustainability, vol. 8, no. 12, p. 1295, 2016.

[66] E. K. Zavadskas, Z. Nunić, Ž. Stjepanović, and O. Prentkovskis, "A novel rough range of value method (R-ROV) for selecting automatically guided vehicles (AGVs)," Studies in Informatics and Control, vol. 27, no. 4, pp. 385-394, 2018.

[67] A. N. Gani and S. N. M. Assarudeen, "A new operation on triangular fuzzy number for solving fuzzy linear programming problem," Applied Mathematical Sciences, vol. 6, no. 11, pp. 525532, 2012.

[68] P. J. V. Laarhoven and W. Pedrycz, "A fuzzy extension of saaty's priority theory," Fuzzy Sets and Systems, vol. 11, no. 1-3, pp. 229241, 1983. 


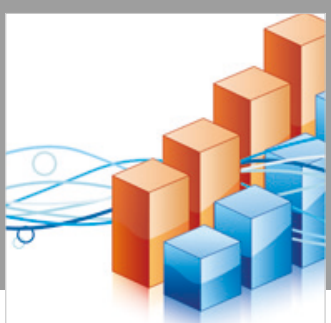

Advances in

Operations Research

\section{-n-m}
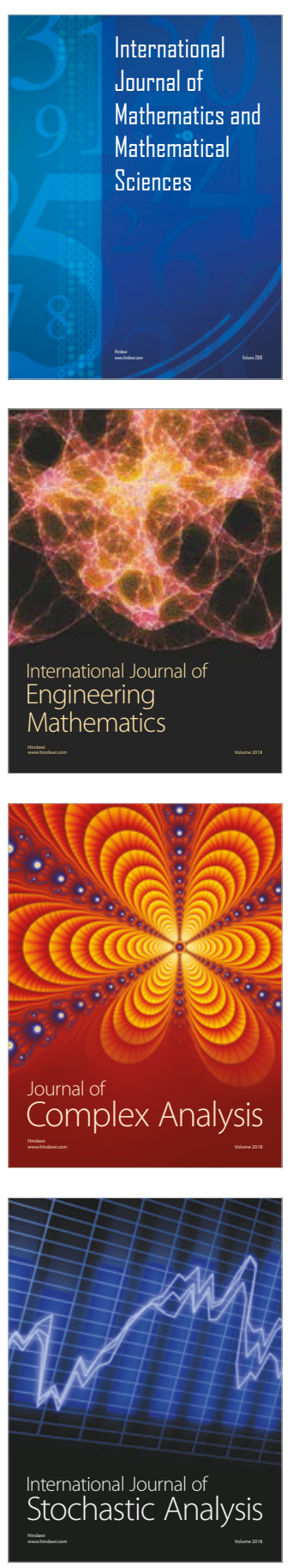
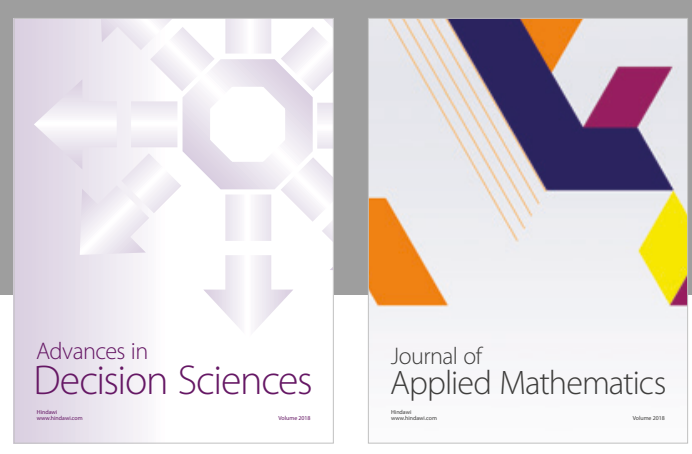

Journal of

Applied Mathematics
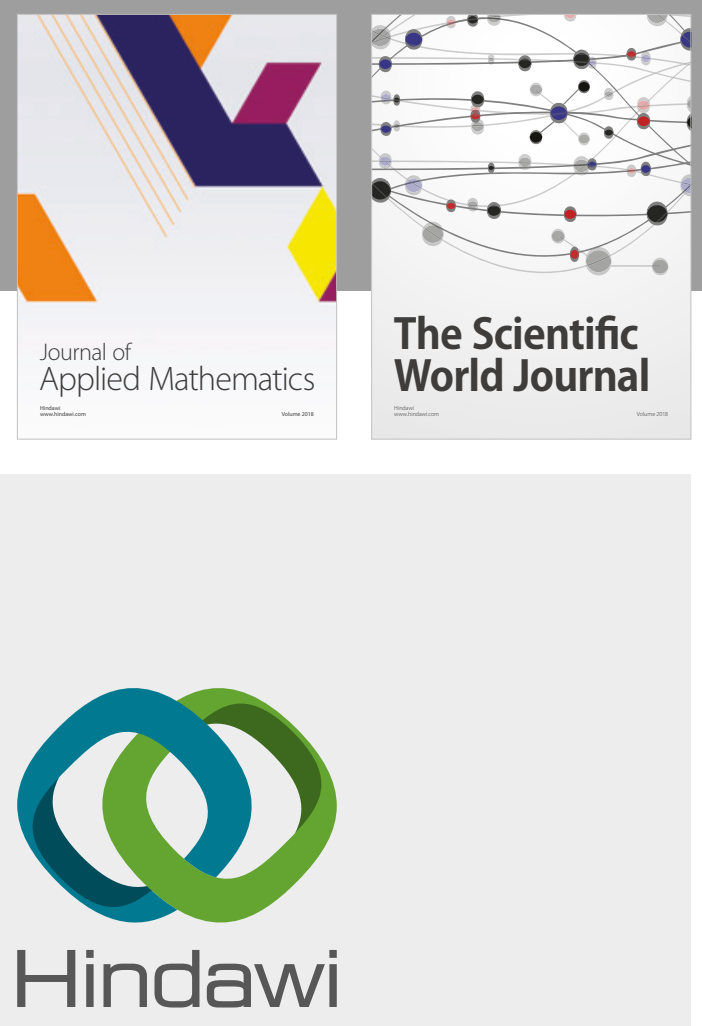

Submit your manuscripts at

www.hindawi.com

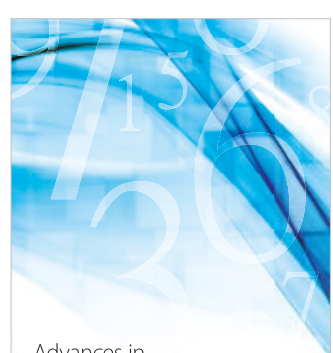

Advances in
Numerical Analysis
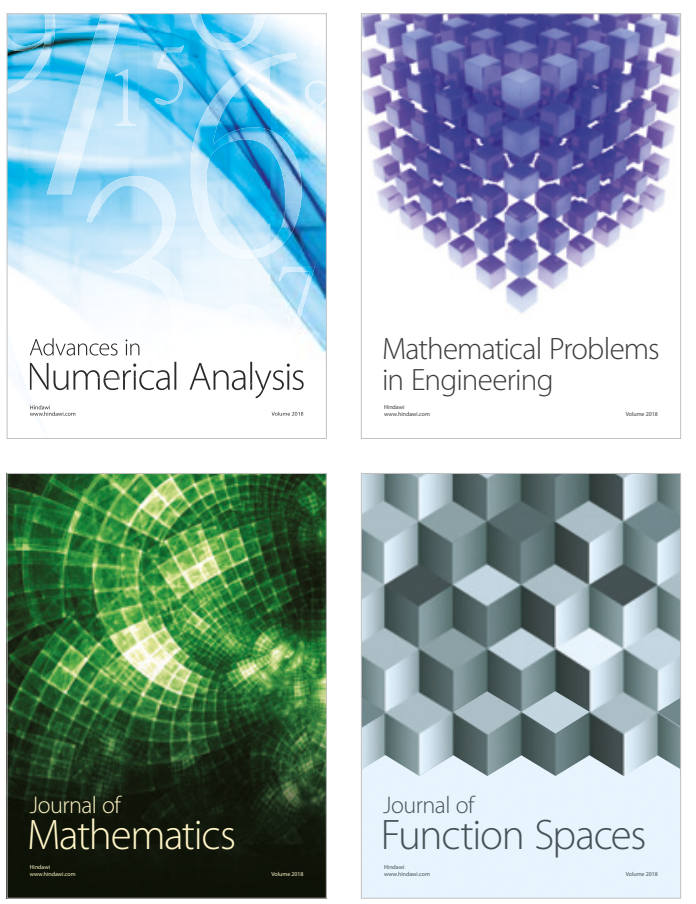

Mathematical Problems in Engineering

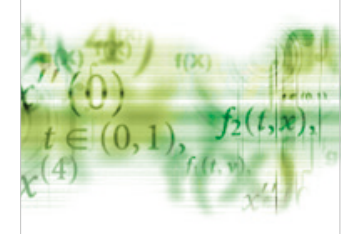

International Journal of

Differential Equations

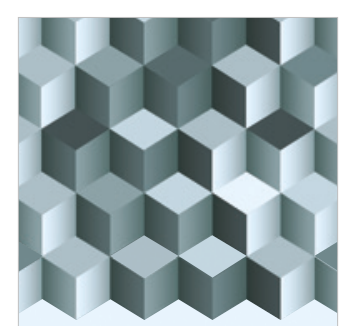

Journal of

Function Spaces

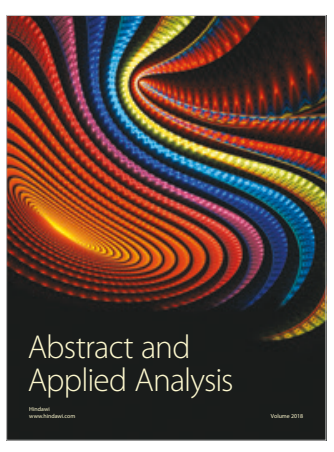

The Scientific

World Journal

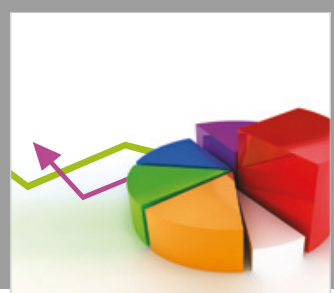

Journal of

Probability and Statistics
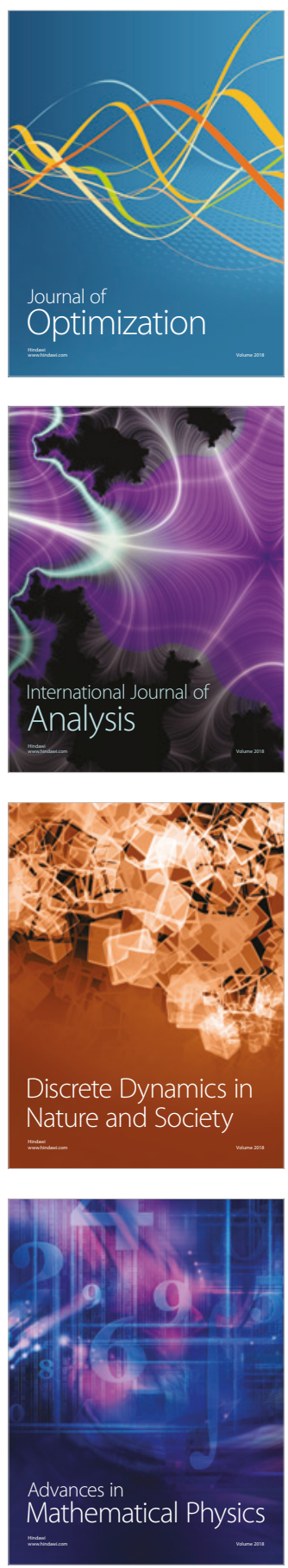